\title{
Enlarged cauda equina nerve roots in cerebrotendinous xanthomatosis
}

\section{Peter Kalina}

Division of Neuroradiology, Department of Radiology, Mayo Clinic, Rochester, USA.

Email: kalina.peter@mayo.edu

Received 20 October 2011; revised 29 November 2011; accepted 8 December 2011.

\begin{abstract}
CXT is a rare inherited autosomal recessive lipid storage disease due to the impaired metabolic pathway of cholesterol secondary to a deficiency in 27sterol hydroxylase, an enzyme in the synthesis of chenodeoxycholic acid (CDCA), a primary bile acid. Abnormal bile acid synthesis leads to elevated plasma Cholestanol (a derivative of cholesterol) accumulation, especially in the lens, central nervous system (CNS) and tendons.
\end{abstract}

Keywords: Cerebrotendinous Xanthomatosis; Cauda Equina; Peripheral Neuropathy

\section{CASE REPORT}

A 41 year old male from Kuwait presented with imbalance, lower extremity weakness and spasticity, difficulty walking and standing. Physical examination revealed hyperreflexia, positive Babinski sign, vibratory sensory loss, bilateral pes cavus, arched feet, hammer toes, valgus deformity of the ankles, atrophic changes of the calves and thighs, Achilles tendon nodules and bilateral cataracts. EMG findings were compatible with peripheral neuropathy. Laboratory examination was notable for elevated plasma cholesterol and increased urine bile alcohol. Serum enzyme studies confirmed the diagnosis of Cerebrotendinous Xanthomatosis (CTX). Magnetic resonance imaging (MRI) of the brain and entire spine with contrast were normal with the exception of diffusely thickened cauda equina nerve roots (Figures 1 and 2).

\section{DISCUSSION}

The classic clinical triad of CTX is Achilles tendon xanthomas, juvenile cataracts and progressive neurological impairment. These clinical hallmarks then prompt a search for increased urine bile alcohol and serum cholestanol. Most severely affected is the cerebellar white matter, optic pathways, brainstem and spinal cord.
Ischemic heart disease, premature atherosclerosis and osteoporosis are also seen. Onset of signs and symptoms is usually in childhood with bilateral cataracts, diarrhea, neurological abnormalities, tendon xanthomas, gait disturbance, epilepsy and polyneuropathy [1].

CNS findings include myelin loss and axonal degeneration. Enzyme defect leads to accumulation of neurotoxic metabolites leading to neuronal loss and axonal degeneration. It remains uncertain whether the pathology is demyelination or neuroaxonal disease with secondary myelin loss [2].

Peripheral neuropathy is manifest clinically as Pes cavus, hammer toes, weakness of small intrinsic muscles of the foot and foot drawn into a claw-like position. EMG confirms the peripheral nerve damage as slow motor/sensory conduction due to demyelinating neuropathy. Sural nerve biopsy confirms demyelinating peripheral neuropathy [3].

MRI findings include symmetric T2 hyperintense lesions in the white matter of the cerebellum and spinal cord and may also be present in the internal capsule, dentate, globus pallidus, substantia nigra, inferior olive as well as adjacent white matter, especially adjacent to basal ganglia and infratentorial, consistent with demyelination. Atrophy may be seen in the cerebrum, cerebellum, brainstem, corpus callosum and cervical cord. MRS findings include decreased NAA reflecting neuronal and axonal damage and increased lactate due to mitochondrial dysfunction from toxic effect of elevated cholestanol and bile alcohol [4].

CDCA replacement therapy slows or reverses disease progression including improving neurological function by suppressing abnormal bile acid synthesis and reducing elevated cholestanol synthesis thus decreasing plasma cholestanol and its deposition [5]. Xanthomas slightly decrease in size. Electrophysiologic studies improve with reduced nerve conduction velocity; slowed somatosensory evoked potentials. Diagnosis and therapy are evaluated by serum cholestanol/cholesterol and urine 


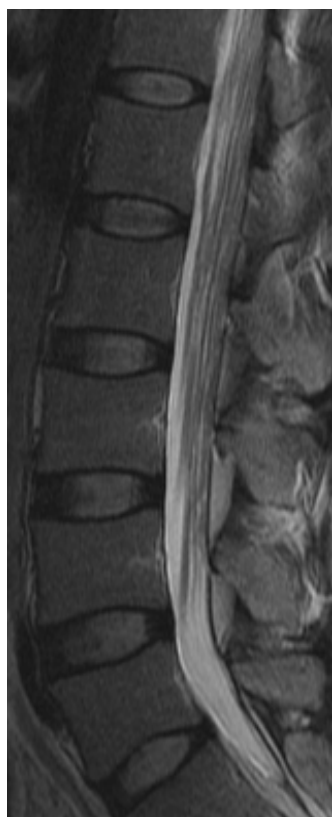

(a)

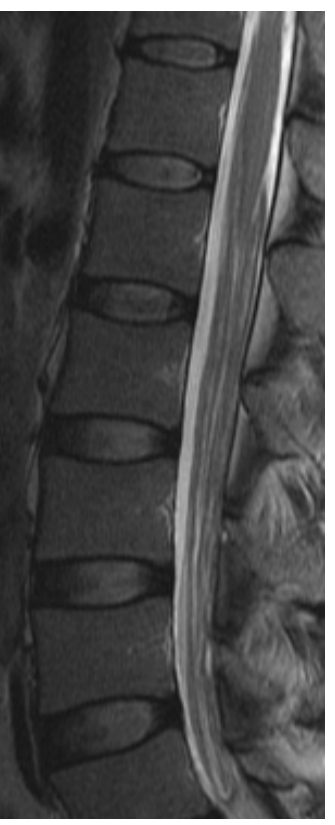

(b)

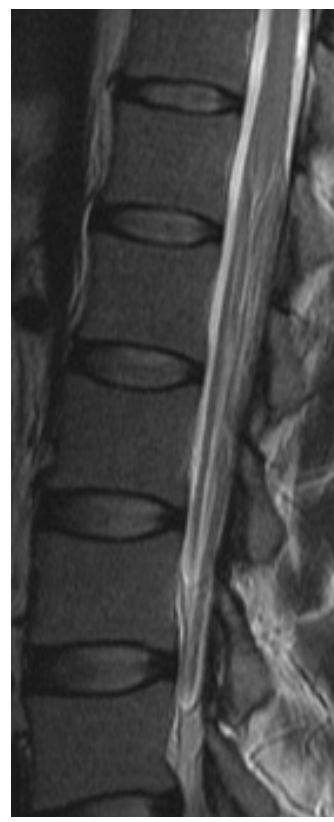

(c)

Figure 1. Sagittal T2 weighted images demonstrating diffusely thickened cauda equina nerve roots.

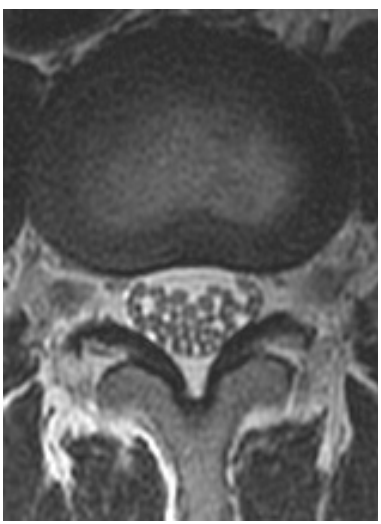

(a)

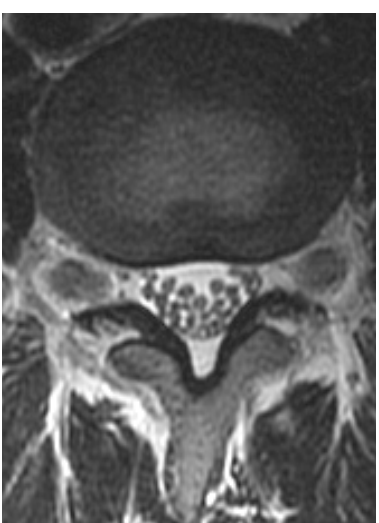

(b)

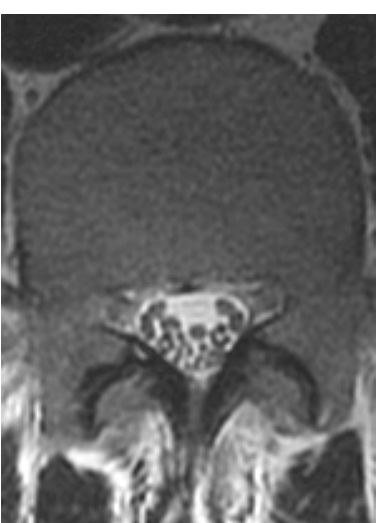

(c)

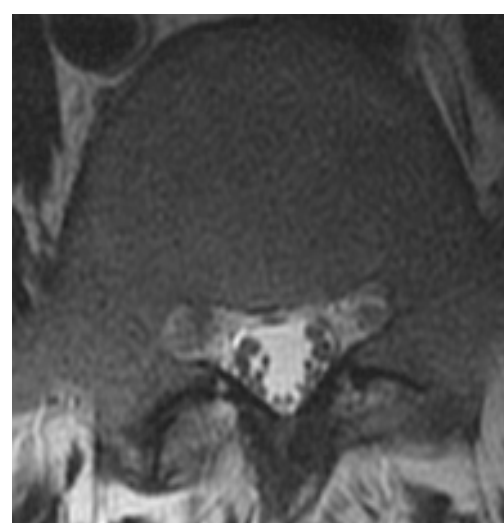

(d)

Figure 2. Axial T2 weighted images confirm the cauda equina nerve root thickening.

excretion bile alcohols.

There are only approximately 300 reported cases of Cerebrotendinous Xanthomatosis. This is likely at least in part due to underdiagnosis and misdiagnosis. While there have been previous descriptions of patients with peripheral neuropathy [6-7], ours represents the only case of CTX where the imaging findings were all entirely normal with the exception of changes consistent with peripheral neuropathy manifest as thickened cauda equina nerve roots. In the appropriate clinical setting, CTX should be included in the differential diagnosis of enlarged cauda equina nerve roots. Conversely, absence of other typical imaging findings of CTX should not exclude the diagnosis in the appropriate clinical setting.

\section{REFERENCES}

[1] Wallon, D., Guyant-Marechal, L. and Laquerriere, A. (2010) Clinical imaging and neuropathological correlations in an unusual case of cerebrotendinous xanthomatosis. Clinical Neuropathology, 29, 361-364.

[2] Pilo, B., De Blas, G. and Sobrido, M. (2011) Neurophysiological study in cerebrotendinous xanthomatosis. Muscle Nerve, 43, 531-536. doi:10.1002/mus.21905

[3] Wang, Z., Yuan, Y. and Zhang, W. (2007) Cerebrotendinous xanthomatosis with a compound heterozygote mutation and severe polyneuropathy. Neuropathology, 27, 62-66. doi:10.1111/j.1440-1789.2006.00739.x

[4] DeStefano, N., Dotti, M., Mortilla, M. and Federico, A. (2001) Magnetic resonance imaging and spectroscopic findings in brain of patients with cerebrotendinous xan- 
thomatosis. Brain, 124, 121-131.

doi:10.1093/brain/124.1.121

[5] Federico, A. and Dotti, M. (2003) Cerebrotendinous xanthomatosis: Clinical manifestations, diagnostic criteria, pathogenesis and therapy. Journal of Child Neurology, 18, 633-638. doi:10.1177/08830738030180091001

[6] Rafiq, M., Sharrack, N., Shaw, P. and Hadjivassiliou, M.
(2011) A neurological rarity not to be missed: Cerebrotendinous xanthomatosis. Practice Neurology, 11,

296-300. doi:10.1136/practneurol-2011-000003

[7] Chen, S., Tsai, N., Chang, C., et al. (2011) Neuromuscular abnormality and autonomic dysfunction in patients with cerebrotendinous xanthomatosis. Bio Medical Central Neurology, 11, 63-72. 